Article de synthèse

\title{
Zonation altitudinale des structures forestières de végétation en Californie méditerranéenne Leur interprétation en fonction des méthodes utilisées sur le pourtour méditerranéen
}

\author{
P. Quezel et M. Barbero
}

Université Aix-Marseille III, centre de Saint-Jérôme, U.A. CNRS 1152, Marseille, France

(reçu le 1er avril 1988; accepté le 8 novembre 1988)

\begin{abstract}
Résumé - Les auteurs proposent pour la région méditerranéenne californienne, une zonation de la végétation basée sur les critères utilisés sur le pourtour méditerranéen. Ils définissent par leurs structures de végétation, respectivement des étages thermo-californien, méso-californien, californien supérieur (ou supracalifornien) montagnard-californien, oro-californien et alti-californien. Les caractéristiques majeures de ces étages et la signification bioclimatique des espèces et des types de végétation sont précisées, essentiellement en fonction des structures forestières. Un certain nombre d'homologies et de différences sont évoquées.
\end{abstract}

forêts - végétation - zonation altitudinale - région méditerranéenne - Californie

Summary - Altitudinal zoning of forest structures in California and around the Mediterranean. A comparative study. The authors suggest for the californian mediterranean region, a zonation of the vegetation based upon the criteria used for the mediterranean margin. Based on the structures of vegetation, they define respectively a thermo-californian level, a meso-californian level, an upper-californian (or supra-californian) level, a mountain-californian level an oro-californian level, and an alti-californian level.

The thermo-californian level includes the southern coastal scrub, some formations with coastal pine and Cupressus, the californian prairie and Quercus douglasii, Q. lobata and Pinus sabiniana forests. The meso-californian level is individualised by the mixed evergreen forest, the sclerophyllous oak woodlands and the chaparral; in cool semi-arid bioclimate, pinyons and junipers are predominant. The upper-californian level is dominated by numerous conifers, and the mountain californian level, by Abies magnifica and Pinus ssp in Sierra Nevada and by Abies concolor and Pinus jeffreyi in transverse ranges. The oro-californian level corresponds to the subalpine forest of Californian authors. Other vegetation structures of azonal significance or not evidently depending on the mediterranean climate are also individualised. The main characteristics of the forest trees are established regarding altitudinal and bioclimatic criteria. In conclusion, homologies and differences between californian and circum-mediterranean forests are mentioned.

forest - vegetation - altitudinal-zoning - Mediterranean - California 

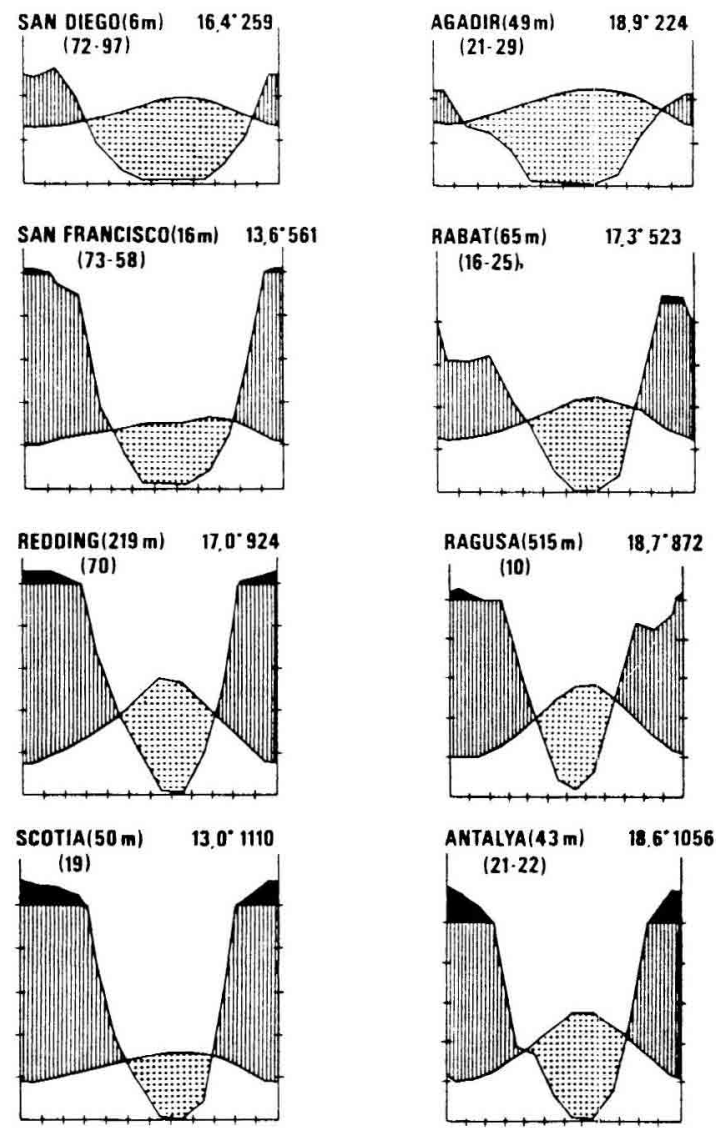

Fig. 1. Diagrammes climatiques de quelques localités en région méditerranéenne et californienne.

\section{Introduction}

La notion de structures altitudinales de végétation (étages de végétation), a fait l'objet de nombreux travaux en région circum-méditerranéenne. Après une phase purement physionomique (Flahault, 1901; Gaussen, 1926) les chercheurs se sont attachés à caractériser les particularités de la région circum-méditerranéenne et ont progressivement défini pour elle, une spécificité à la fois biogéographique et écologique, qui a débouché sur des schémas actuellement adoptés, à quelques nuances terminologiques près, par les phytogéographes (Ozenda, 1975; Quezel, 1974; Barbero et Quezel, 1981; RivasMartinez et Arnaiz, 1984). En Californie méditerranéenne en revanche, les auteurs en sont restés à une interprétation basée sur l'aspect global des grandes structures de végétation et ne tenant pas compte a priori, des particularités bioclimatiques et éco-physiologiques régionales. L'un de nous (Quezel et Schevock, 1982), avait déjà tenté d'établir un certain nombre de corrélations entre les étages de végétation reconnus sur le pourtour méditerranéen et 
ceux de Californie, mais il nous a paru intéressant de pousser plus avant cette mise en parallèle. II convenait, en premier lieu, de chercher à préciser les correspondances possibles qui pouvaient être trouvées entre les étages de végétation de ces deux régions climatiquement très proches (Koppen, 1936; Ackerman, 1941; Aschmann, 1973), mais aussi physionomiquement homologues.

Sans vouloir entrer ici dans de longues descriptions, il convient toutefois de souligner les analogies complètes qui apparaissent, en particulier au niveau des "Diagrammes climatiques" établis selon la méthode de Walter (in Walter, Harnickell et Mueller-Dombois, 1975) elle-même directement dérivée de celle créée par Bagnouls et Gaussen (1953) entre les diverses situations bioclimatiques existant en région circum-méditerranéenne et en Californie. A titre indicatif, nous avons fait figurer dans la Fig. 1 quelques exemples particulièrement évocateurs.

Nous avons donc tenté, en utilisant les critères retenus sur le pourtour méditerranéen, de rattacher les principales structures forestières reconnues en Californie méditerranéenne, à la zonation altitudinale classiquement définie sur le pourtour méditerranéen. Cette tentative est illustrée sur la carte de végétation que nous publions dans ce travail (Fig. 2).

Cette approche nous a paru par ailleurs nécessaire, à un moment où les essences californiennes sont de plus en plus utilisées pour les reboisements en région circum-méditerranéenne. En effet, il est indispensable de connaître les exigences bioclimatiques et altitudinales que présentent ces espèces dans leur région d'origine, afin de les introduire dans des conditions écologiques analogues. D'autre part, la mise en place en région méditerranéenne californienne d'une zonation identique à celle qui est retenue en région circum- méditerranéenne, devrait faciliter considérablement le choix non seulement des espèces, mais des races ou des écotypes les mieux adaptés pour être utilisés sur le pourtour méditerranéen.

\section{La région méditerranéenne californien- ne (Fig. 3)}

La région méditerranéenne californienne peut être définie par son climat comme nous venons de lindiquer, mais aussi par sa flore et par sa végétation. Cette région floristique a été étudiée par de nombreux auteurs et en particulier par Raven et Axelrod (1977); elle ne correspond que très approximativement à l'état de Californie dont elle exclut en particulier les marges désertiques orientales, et pénètre en revanche, au nord en Orégon et au sud au Mexique dans la province de Baja California. Nous n'insisterons pas davantage ici sur les caractères de cette région floristique et renvoyons pour cela le lecteur à l'article cité ci-dessus.

La végétation californienne, et tout spécialement ses structures forestières, sont bien connues, comme le montre la synthèse publiée par Barbour et Major (1977) à laquelle est jointe une carte de la végétation naturelle réalisée par Kuchler. Toutefois, les divers articles constituant cet ouvrage collectif, ne permettent pas de se faire une idée précise de la zonation altitudinale de la végétation, car ils sont le plus souvent consacrés à un type physionomique, à un genre ou à un groupe d'espèces affines, dont l'extension altitudinale n'est pas toujours a priori significative du point de vue qui nous intéresse ici. En effet, en région circum-méditerranéenne, ces deux types de situation s'inscrivent très souvent sur plusieurs étages et séries de végétation (cas de Quercus rotundifolia au Maroc par exemple, in Barbero, 


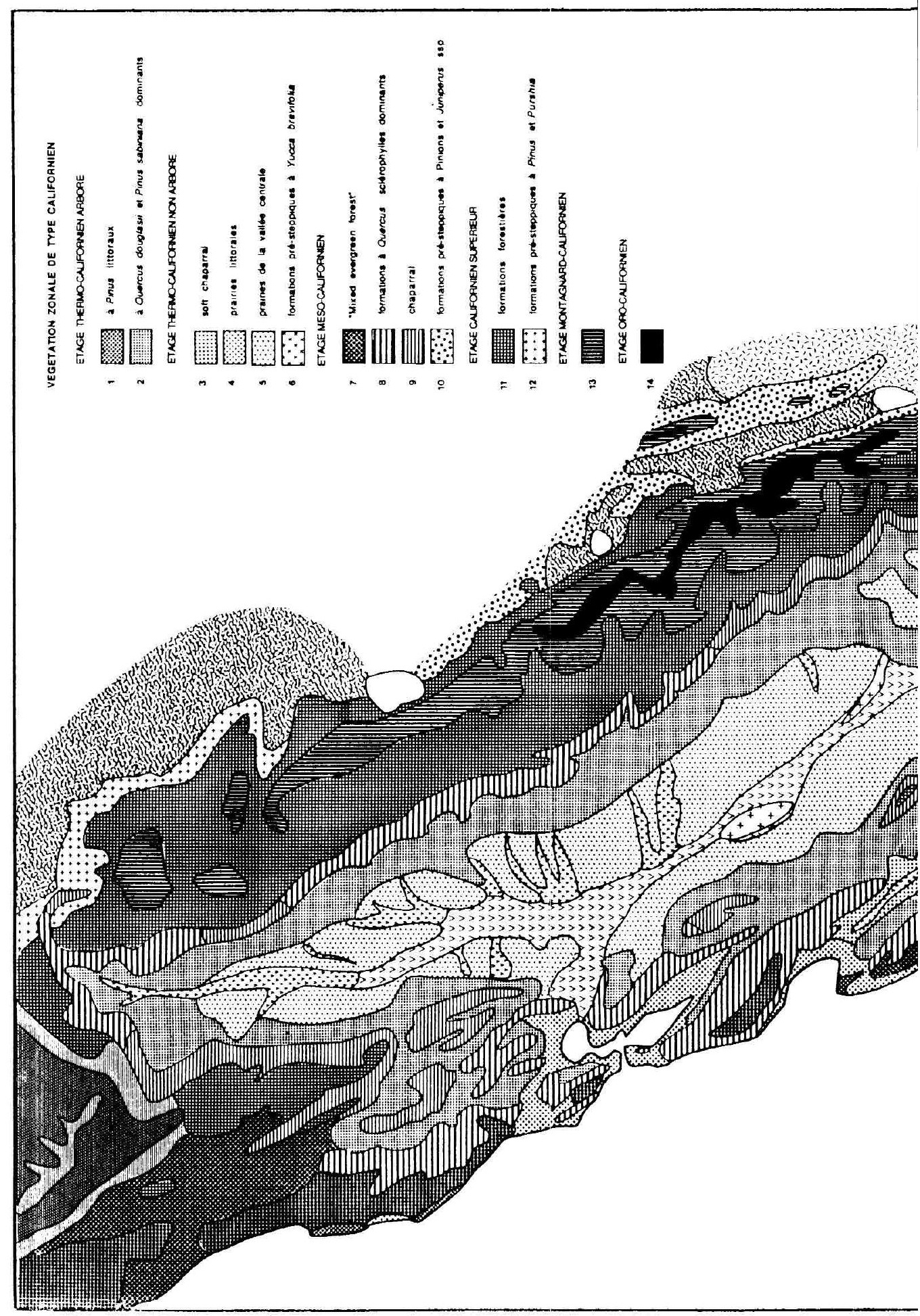




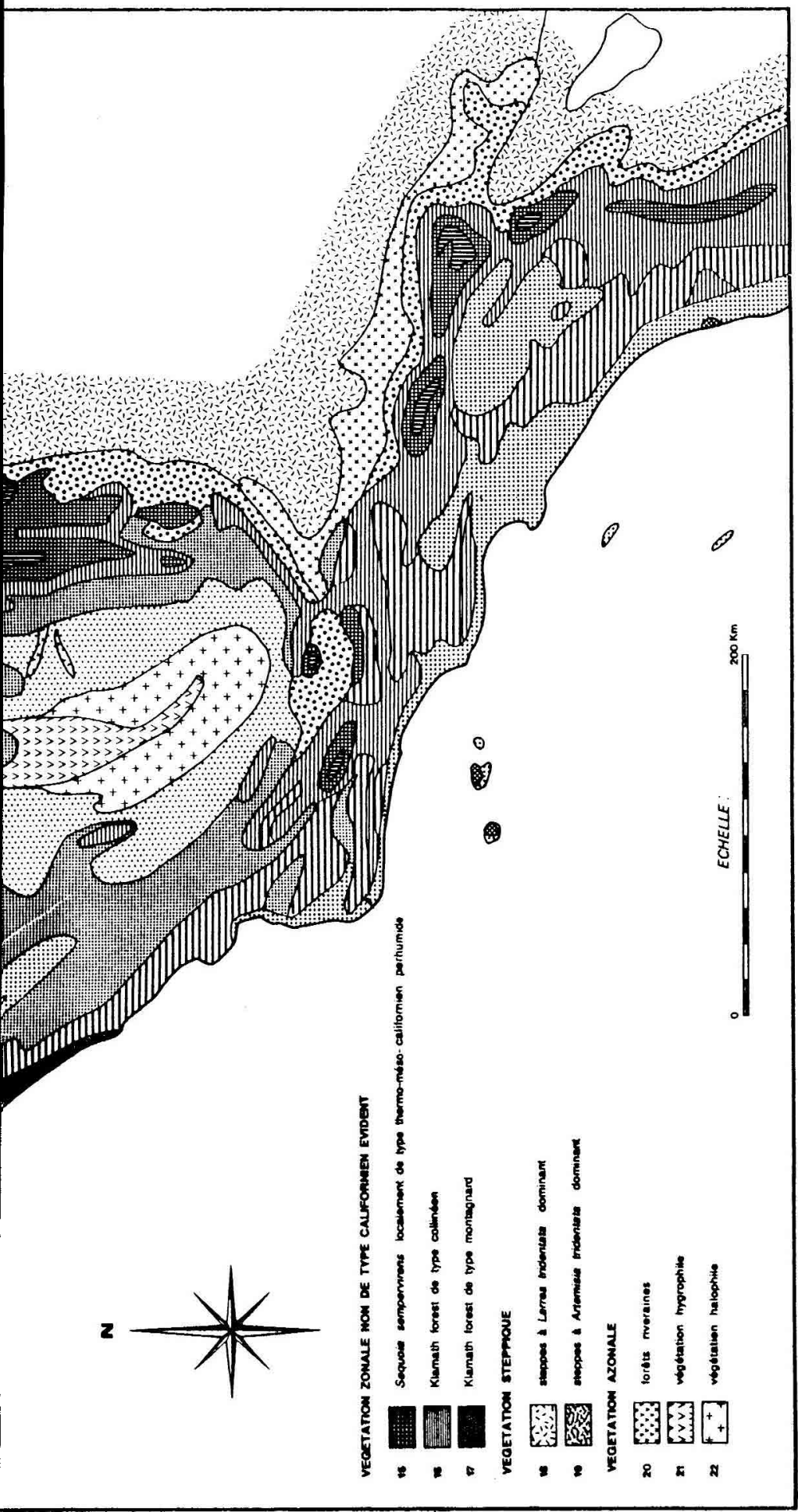

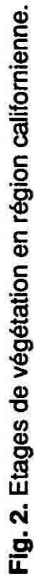


Quezel, Rivas-Martinez, 1981). La seule tentative de classification altitudinale de la végétation en Californie, selon les critères utilisés en région circum-méditerranéenne, est celle de Quezel et Schevock indiquée ci-dessus. Sans entrer dans une interprétation trop rigide, en raison de notre connaissance insuffisante du terrain, il paraît possible de dresser les grandes lignes de cette mise en parallèle.
II ne semble pas utile de modifier pour la Californie, les critères et la terminologie établis en région circum-méditerranéenne. Toutefois, il parait plus significatif d'utiliser une terminologie mieux adaptée aux critères biogéographiques et de distinguer ici les étages thermo-californien, méso-californien, californien supérieur (ou supracalifornien), montagnard-californien, orocalifornien et alti-californien.

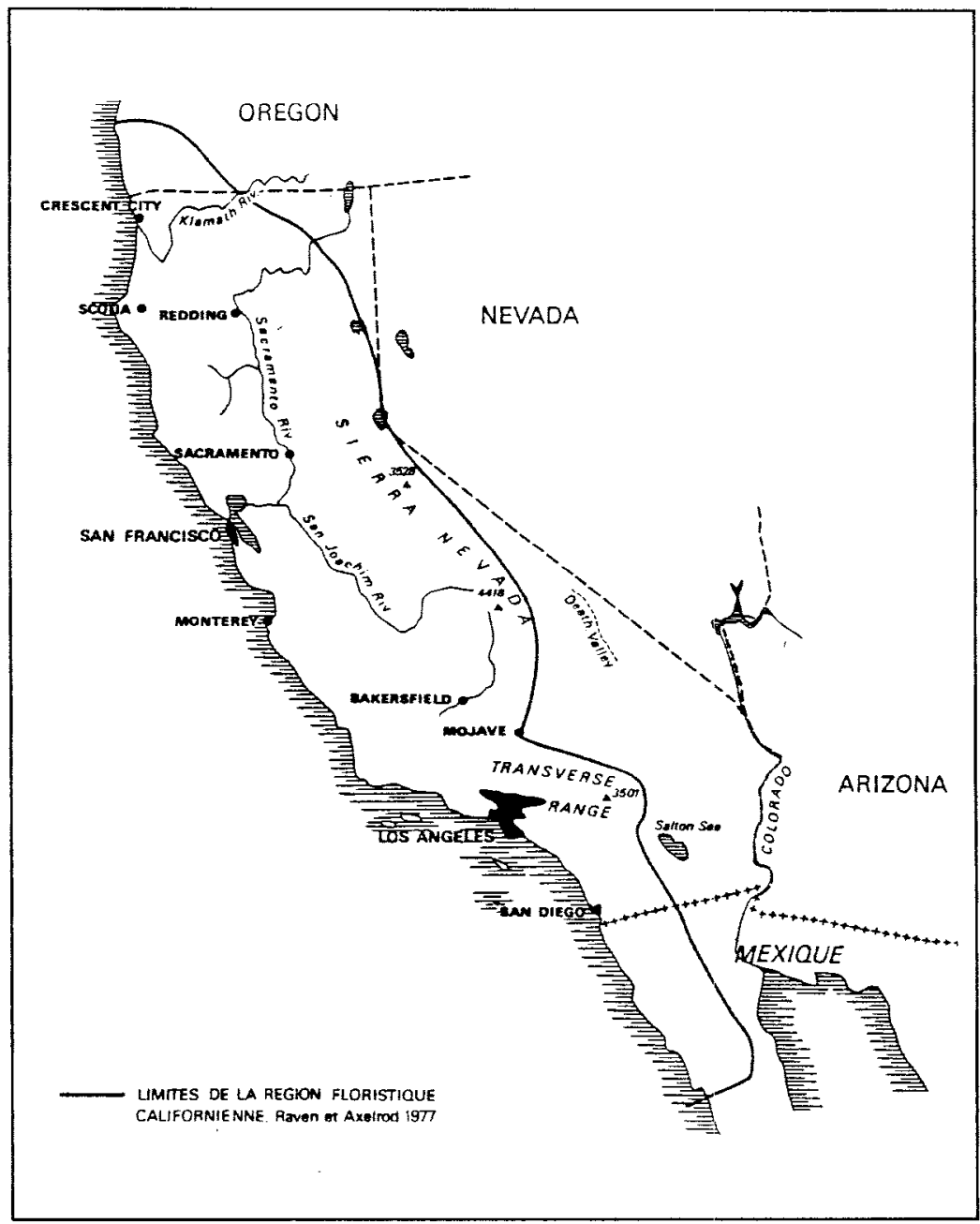

Fig. 3. La région méditerranéenne californienne. 
Cette interprétation pose un problème qu'il est bon d'évoquer : les structures de végétation incluses sous ces vocables se rattachent à une compréhension du climat méditerranéen qui est celle des auteurs européens (Emberger, 1930, 1945; Daget, 1977; Quezel et Barbero, 1985) plutôt que celle plus restrictive de divers auteurs californiens (cf. à ce propos Aschmann 1984) qui, si elle était retenue, exclurait des régions méditerranéennes la majeure partie du méso-méditerranéen ou du mésocalifornien, et la totalité des étages susjacents. De même le rôle du facteur "vent", de toute évidence, plus accusé en région circum-méditerranéenne, ne sera pas évoqué ici.

En fait, dans ce travail, nous envisagerons à la fois "The californian floristic province" et "The sierran floristic province" dont les caractères bioclimatiques peuvent sans problèmes majeurs être mis en parallèle avec ceux de la région circumméditerranéenne, telle qu'elle est généralement comprise. Nous en exclurons ou évoquerons succinctement "The pacific north-west floristic province" bien que certaines parties de celle-ci soient encore, théoriquement au moins, sous régime climatique méditerranéen, mais où cependant la période de sécheresse estivale n'est pas évidente, et aussi les zones subdésertiques orientales où il n'existe pas de climax forestier ou même arboré.

Un autre problème important qu'il convient d'évoquer avant de définir les étapes de végétation, tient au fait qu'en région méditerranéenne de Californie, les substrats sont constitués à peu près exclusivement par des roches non calcaires, et ceci, contrairement à ce qui se passe en région circum-méditerranéenne. Cette particularité, évoquée du point de vue floristique par Raven et Axelrod (1977) est importante, et ces auteurs arrivent à peine à indiquer quelques espèces inféodées aux rares affleurements de cal- caire ou de dolomie, alors que les roches ultra-basiques largement présentes en Californie, possèdent de nombreuses espèces endémiques et notamment divers Cupressus et Quercus durata.

II faut convenir que ces particularités limitent considérablement, au moins du point de vue théorique, les comparaisons qui peuvent être établies entre les exigences écologiques des essences forestières californiennes et circum-méditerranéennes. Toutefois, cette situation est imposée par la nature même des substrats, et diverses tentatives d'introduction d'espèces californiennes réputées calcifuges, dans des reboisements sur divers types de roches en région circum-méditerranéenne, ont fourni d'excellents résultats. Il convient donc de relativiser ces différences, et de ne pas exclure a priori en région circum-méditerranéenne des substrats calcaires, les essences californiennes qui ne colonisent pas dans leur habitat naturel ce type de substrat. Le succès des reboisements de pin laricio de Corse ou de Calabre sur des sols calcaires en France méditerranéenne est d'ailleurs une illustration très claire de ce phénomène.

\section{L'étage thermo-californien}

Sa végétation a été fort malmenée et, bien souvent, ne subsistent à son niveau que des formations de dégradation. Il est possible de la reconnaître en zone littorale, dans la vallée centrale et sur le revers oriental des chaînes californiennes.

\section{En zone littorale}

Divers types de paysage se rapportent, en zone littorale, à cet étage.

- "The southern coastal scrub" (Mooney, 1977), ou "coastal sage scrub" (Clements, 1920), ou "soft chaparral" (Cooper, 1922). 
Cette formation végétale très particulière, dominée par Artemisia californica, divers Salvia, Baccharis, Erigonum, Haplopappus, présente une activité phénologique axée sur l'hiver et le printemps et se rapproche de ce point de vue des phryganes ${ }^{1}$ de la région méditerranéenne orientale (Quezel, 1981), bien que l'aspect soit différent et plus proche des matorrals méditerranéens à Cistus. Du point de vue climatique (Hanes, 1981), les précipitations restent faibles (300 et $500 \mathrm{~mm}$ en moyenne) et les moyennes des minima des températures du mois le plus froid, élevées de 5 à $8^{\circ} \mathrm{C}$ ), ce qui permet de situer cette végétation dans un étage thermo-méditerranéen tempéré et chaud. Le problème majeur du point de vue dynamique est ici celui d'un hypothétique climax forestier ou du moins arboré. Il est actuellement bien difficile de proposer une solution étant donné l'absence quasi totale d'arbres à cet étage.

- La prairie littorale (Heady et al., 1977) remplace le "soft chaparral" au nord de Monterrey; elle est dominée par les graminées vivaces, le plus souvent autochtones (Festuca idahoensis, F. rubra, Danthonia californica, etc.) et est associée à des broussailles à Baccharis ssp., Artemisia ssp. Elle représente probablement une formation secondaire, due aux incendies et au pâturage.

- Certaines formations à pins liftoraux (closed-pine) et à Cupressus qui sont de plus en plus résiduelles vers le sud de la Californie, pourraient représenter, au moins localement, les formations potentielles forestières du "soft chaparral" ou de la prairie littorale. Elles sont, par ailleurs, morphologiquement et écologiquement vicariantes des formations circum-méditerranéennes de cet étage à Cupressus et

1. Mattoral bas de type thermo-méditerranéen décidu en été.
Pinus du groupe halepensis-brutia. En fait, en Californie comme en région circumméditerranéenne, ces arbres se développent souvent hors de l'étage thermo-méditerranéen, et dans cette dernière région, sont largement présents au méso-méditerranéen, où ils jouent un rôle paraclimatique vis-à-vis des structures à Quercus sclérophylles: Pinus halepensis pour $Q$. ilex et $Q$. rotundifolia, $P$. brutia pour $Q$. calliprinos, $P$. pinaster pour $Q$. suber. II serait intéressant de préciser s'il en est de même en Californie, ce qui est d'ailleurs probable au moins pour certains d'entre eux, notamment $P$. attenuata. En revanche $P$. radiata, $P$. contorta et $P$. toreyana se localisent en peuplements fortement résiduels dans cet étage ainsi d'ailleurs que plusieurs des 10 Cupressus californiens (Vogl et al., 1977) C. macrocarpa, C. goveniana en particulier.

\section{Dans la vallée centrale}

L'étage thermo-californien est constitué essentiellement par la prairie californienne, où s'installent localement divers Quercus à feuilles caduques.

- Jusqu'à 200-400 m, en fonction surtout des expositions, se développe la "prairie californienne" (H.-F. Heady, 1977) essentiellement constituée de graminées annuelles ou vivaces où les espèces introduites de la région méditerranéenne sont souvent dominantes. Dans cette zone apparaissent, rotamment aux environs de Bakersfield des éléments nettement subdésertiques, en général de souche tropicale. En revanche, les formations riveraines, extrêmement malmenées, sont dominées par Quercus lobata, espèce caducifoliée, dont de magnifiques individus existent encore çà et là.

- Entre 200-400 m et 600-700 m en exposition nord et 900-1000 m environ en exposition sud, la prairie californienne persiste, mais se couvre inégalement, en 
fonction des critères climatiques édaphiques et surtout anthropiques, d'un piqueté arborescent où Quercus douglasii (lui aussi caducifolié) domine, associé à l'horizon supérieur de cette zone à Aesculus californica, et localement, à Pinus sabiniana. $Q$. lobata est toujours présent dans les ravins, les dépressions et sur sols à bon bilan hydrique. A la frange supérieure de cette zone, surtout dans les thalwegs, apparaissent déjà les chênes sclérophylles ( $Q$. wislizenii surtout).

Sur le revers oriental des chaînes californiennes

L'étage thermo-californien paraît ici mal représenté en raison des critères climatiques. En effet, les formations à "pinyons» 2 de type méso-californien entrent souvent directement en rapport avec le «scrub" désertique à Larrea tridentata. Localement au moins, certaines formations clairsemées de type pré-steppique constituées surtout par Juniperus californica, Ephedra nevadensis, et souvent associés à Yucca brevifolia, paraissent devoir être rattachées à cet étage de végétation.

\section{L'étage méso-californien}

Comme l'étage méso-méditerranéen, il est dominé par les Quercus sclérophylles, plus ou moins envahis par les Conifères. Les chaparrals typiques répondent également le plus souvent à cet étage. Le méso-californien présteppique enfin est très bien représenté.

Les auteurs californiens distinguent, en général "the mixed evergreen forest" et "the Oak woodland", dont la place dans cet étage mérite d'être discutée.

2. Nom local et général des pins à grosses graines comestibles.
- "The mixed evergreen forest" (Sawyer, Thornburg et Griffin, 1977) apparaît tout au long des chaînes côtières, et dans des conditions écologiques qui paraissent se rapporter au méso-californien humide. Plusieurs types ont été distingués du nord au sud, mais ils présentent tous en commun des Quercus sclérophylles associés à des Conifères, à Umbellularia californiaca, à Lithocarpus densiflora, le genre Arbutus est également présent. Parmi les conifères, Pseudotsuga menziesii, Pinus coulteri, $P$. jeffreyi, Sequoia sempervirens, Abies bracteata, Pseudotsuga macrocarpa peuvent apparaitre, selon les localités. Parmi les Quercus, les types sempervirens dominent: $Q$. chrysolepis et $Q$. agrifolia surtout, mais laissent une certaine place aux types caducifoliés en particulier vers le nord : $Q$. kelloggi, $Q$. garryana. Ces formations très complexes débordent très certainement du méso-californien, en particulier vers le bas, surtout en position juxta-littorale, mais il est encore impossible de fournir de plus amples informations. Soulignons également que l'on peut les mettre en parallèle de façon évidente avec les laurisylves canariennes (Santos-Guerra, 1984), beaucoup moins riches du point de vue floristique, mais qui traduisent une physionomie souvent proche, et surtout, des conditions écologiques fort voisines. Rappelons que la laurisylve canarienne se situe à la charnière entre les étages thermo- et méso-canariens!

- "The Oak woodland" des auteurs californiens (Griffin, 1977) comprend à la fois des formations sciérophylles et des formations caducifoliées. Nous avons parlé de ces dernières plus haut, car elles s'incluent essentiellement au thermo-californien. Les formations sempervirentes regroupées sous ce vocable correspondent en fait à des structures dominées par Quercus chrysolepis, $Q$. wislizenii et $Q$. agrifolia, mais plutôt en bioclimat sub- 
humide, ce qui en exclut de nombreuses essences exigeantes. Ces formations sont très semblables aux chênaies sclérophylles circum-méditerranéennes à $Q$. ilex, $Q$. rotundifolia ou $Q$. calliprinos. Des intermédiaires existent bien sûr entre "the mixed evergreen forest" et "the Oak sclerophyllous woodland".

Dans la chaîne péninsulaire, à l'étage méso-californien Quercus agrifolia s'associe au singulier semi-sempervirent $Q$. engelmanii (Snow, 1972). Cet arbre joue en Californie méridionale, un rôle très comparable à celui des Quercus semisempervirents du groupe Aegylops (Quezel et Bonin, 1980) en Méditerranée orientale, et constitue comme ceux-ci (Akman, Barbero et Quezel, 1978), soit des forêtsparcs très clairsemées à strate herbacée bien développée, soit des forêts denses.

- Le chaparral (Hanes, 1977) représente une structure très caractéristique du méso-californien, dont elle n'est d'ailleurs pas exclusive. Elle trouve là, toutefois, son développement optimal et constitue l'équivalent très précis des matorrals circumméditerranéens. Divers types floristiques ont été définis notamment ceux à Adenostoma fasciculatum, à Ceanothus, à Arctostaphyllos, en fonction surtout de leur répartition géographique, soit à Quercus sclérophylles de petite taille, notamment à $Q$. dumosa ou $Q$. turbinella. La plupart des auteurs californiens ont tendance à considérer les chaparrals comme des formations climaciques, opinion qui mérite au moins d'être discutée (Quezel, 1979). Essentiellement localisés au méso-californien subhumide, leur évolution vers des structures dominées par les Quercus sclérophylles ou divers Pinus nous paraît, au moins localement, envisageable en dehors des zones soumises à des éclosions trop rapprochées de feux naturels ou provoqués.

Les paysages de chaparral en Californie ne sont d'ailleurs, en aucune façon, liés à l'étage méso-californien; nous les avons déjà signalés au thermo-californien ("soft chaparrals"), et ils pénètrent très largement dans les étages sus-jacents ("montane chaparral") où ils sont alors en corrélation évidente avec les structures forestières (Quezel et Taylor, 1984).

- Les formations méso-californiennes présteppiques constituent un paysage répandu en Californie. Elles se localisent en bioclimat semi-aride frais essentiellement, et correspondent à des structures très particulières de végétation, surtout représentées sur le revers oriental des chaînes californiennes, c'est-à-dire au contact des grandes zones subdésertiques du Mojave ou du Sonoran. Les éléments les plus significatifs sont diverses espèces de Pinus à grosses graines, généralement regroupés sous le nom de "pinyons", et qui s'étendent par ailleurs vers l'est bien au-delà des limites de la région méditerranéenne californienne. Ces pins, Pinus monophylla, $P$. quadrifolia et $P$. edulis surtout, apparaissent en fonction de critères géographiques et écologiques et s'associent à divers Juniperus (J. californica, $J$. osteosperma) et à divers chaméphytes dont plusieurs participent aux paysages de chaparral et où les Quercus sclérophylles bas sont localement présents, $Q$. turbinella et $Q$. dumosa en particulier. Lorsque les altitudes s'abaissent ces formations passent progressivement à celles du thermo-californien présteppiques dominées (cf. supra) par les Juniperus et les Ephedra. Le parallèle est grand avec les ensembles semi-arides de l'Atlas (Quezel et Barbero, 1981) à Juniperus phoenicea, J. oxycedrus et Ephedra ssp.

\section{L'étage californien supérieur}

Contrairement à ce qui se passe en région circum-méditerranéenne, où l'étage correspondant est dominé par les Quercus 
sclérophylles sur le revers méridional du bassin et les Quercus caducifoliés sur le revers septentrional (Quezel et Bonin, 1980); ce sont ici les conifères qui constituent l'élément physionomique majeur. Certes, en Californie, localement Quercus kelloggii, voire $Q$. chrysolepis peuvent jouer un rôle appréciable, mais cet étage correspond essentiellement à "the lower montane coniferous forest" des auteurs californiens, végétation essentiellement développée sur la Sierra Nevada (Rundel, Parson et Gordon, 1977) et sur les chaînes transverses et péninsulaires (Thorne, 1977). Du point de vue altitudinal elle apparait suivant la latitude et l'exposition entre 1100 et 2100 en Sierra Nevada et 1 200-2 $000 \mathrm{~m}$ sur les chaînes transverses et péninsulaires.

Les structures de végétation sont relativement complexes, mais il est possible de distinguer schématiquement, en fonction des situations géographiques deux horizons.

\section{En Sierra Nevada méridionale et centrale}

A partir de 1 100-1 $200 \mathrm{~m}$ en versant nord et 1400-1 $500 \mathrm{~m}$ en versant sud, les conifères deviennent prédominants. Un ensemble relativement homogène s'étend jusque vers $2100-2300 \mathrm{~m}$. Les représentants du genre Quercus n'en sont pas exclus; les chênes sclérophylles et $Q$. chrysolepis, en particulier, apparaissent surtout en versant sud et sur les substrats superficiels, alors que $Q$. kelloggii constitue de son côté des formations mixtes avec les Pins surtout à l'horizon inférieur de cette zone qui s'étend jusque vers 1600-1 $700 \mathrm{~m}$. Pinus ponderosa est largement dominant associé à Quercus kelloggi mais aussi à Calocedrus decurrens et à Pinus jeffreyi.

L'horizon supérieur est constitué par les mêmes conifères, Pinus ponderosa, Pinus jeffreyi et Calocedrus decurrens sur- tout, associés à Pinus lambertiana. Toutefois, en situation mésophile Abies concolor devient dominant, alors qu'en position méso-hygrophile apparaît Sequoiadendron giganteum.

Sur les chaînes transverses, le schéma est à peine différent

A partir de $1300-1400 \mathrm{~m}$ en moyenne, les conifères dominent. II s'agit surtout de Pinus ponderosa et de Pinus jeffreyi. Calocedrus est abondant; Pseudotsuga macrocarpa mais aussi Pinus coulteri peuvent être présents. Comme le fait remarquer Thorne (1977), sur les chaînes transverses, c'est Pinus jeffreyi qui est le plus répandu, en particulier en versant nord; ce Pin pénètre d'ailleurs largement à l'étage montagnard. Deux horizons peuvent être distingués ici encore :

- un horizon inférieur où Pinus ponderosa est généralement abondant, et où se rencontre encore Quercus chrysolepis, mais aussi $Q$. kelloggii. Toutefois, ce chêne caducifolié est ici bien moins fréquent que sur la Sierra Nevada. Pinus coulteri et Pseudotsuga macrocarpa peuvent être localement présents;

- un horizon supérieur, en général à partir de 1600-1 $700 \mathrm{~m}$, où Pinus jeffreyi domine et s'associe, surtout en versant nord à des éléments plus alticoles, tels que Abies concolor et Pinus lambertiana. II atteint en général $2000 \mathrm{~m}$.

\section{Sur les chaînes péninsulaires}

L'étage californien supérieur, assez mal développé, s'étend entre $1400-1500 \mathrm{~m}$ et les sommets; il est constitué par des peuplements souvent disjoints de Pinus jeffreyi ; les chênes sclérophylles sont encore présents.

Les structures de végétation que nous venons d'évoquer appartiennent essentiellement au bioclimat humide et sub-humide 
froid (Harvay, 1980; Quezel et Schevock, 1982); elles occupent essentiellement les façades occidentales et méridionales des chaînes (cismontanous); elles pénètrent largement sur les versants ouest et nord (transmontanous) où toutefois, une diminution rapide des précipitations détermine l'individualisation de structures présteppiques en bioclimat semi-aride froid surtout, dont la constitution nous est mal connue. Sur les chaînes transverses, il s'agit essentiellement de formations à Pinus jeffreyi et/ou à Juniperus occidentalis, où Pinus monophylla est encore présent.

- Sur le revers nord-occidental de la Sierra Nevada, ces formations remplacent les forêts claires de type présteppique dominées par Pinus ponderosa. Juniperus occidentalis et Purshia tridentata où Abies concolor peut apparaître. Ces formations doivent elles aussi se rapporter à un étage méditerranéen supérieur présteppique (semi-aride froid) et cèdent progressivement la place vers l'est à des steppes arborées à Artemisia tridentata et Juniperus occidentalis puis aux steppes à Artemisia tridentata.

\section{L'étage montagnard californien}

II succède au précédent dans les diverses situations évoquées ci-dessus, sauf sur les chaînes péninsulaires où il fait défaut. II s'étend approximativement suivant les conditions géographiques et écologiques entre 2000 et $2800 \mathrm{~m}$. Les structures de végétation qui le caractérisent correspondent aux "upper montane coniferous forests" des auteurs californiens. En Sierra Nevada, Abies magnifica occupe la place principale. II peut encore s'associer sur ses limites inférieures aux arbres caractéristiques de l'étage californien supérieur qui néanmoins disparaissent rapidement et sont remplacées localement par Pinus contorta subsp. murrayana en situation mésoxérophile, voire par Pinus monticola. Pinus jeffreyi reste toutefois présent sur les substrats superficiels, alors que Populus tremuloides apparaît très épisodiquement sur des sols plus ou moins hydromorphes.

Sur les chaînes transverses la situation n'est guère différente :

- en situation mésophile, la forêt climacique est dominée par Abies concolor et Pinus lambertiana; $P$. jeffreyi reste cependant généralement présent, ainsi que Calocedrus decurrens;

- en situation méso-xérophile, $P$. jeffreyi constitue l'espèce dominante et $P$. contorta subsp. murrayana peut apparaître, surtout aux hautes altitudes;

- en situatiorı xérophile, c'est essentiellement la forêt claire à Juniperus occidentalis ssp. ausiralis qui se développe (Thorne, 1977); localement les pins, voire Abies concolor sont présents, alors que le sous-bois est constitué par une végétation de type chaparral où dominent les représentants des genres Arctostaphylos, Eriogonum, Cercocarpus, Ceanothus, etc.

\section{L'étage oro-californien}

II correspond à l'étage des "subalpine forests" des auteurs californiens qui est surtout bien développé sur la Sierra Nevada (Rundel et al., 1977), et s'étend entre $2700-2900 \mathrm{~m}$ et la limite supérieure des arbres qui se situe ici vers $3500-3600 \mathrm{~m}$. Ces forêts claires sont constituées essentiellement par Pinus albicaulis, $P$. balfouriana, $P$. flexilis, $P$. monticola et Tsuga mertensiana. La valeur écologique et biogéographique de ces diverses espèces reste en grande partie à préciser. Sur les chaînes transverses Pinus contorta subsp. murrayana et Pinus flexilis sont les éléments les plus significatifs, mais ils res- 
tent souvent associés à Abies concolor et Juniperus occidentalis. En Sierra Nevada, comme sur les chaines transverses, le sous-bois est essentiellement constitué par des buissons le plus souvent à feuillage persistant.

\section{L'étage alti-californien}

C'est avec "the alpine belt" des auteurs californiens (Major et Taylor, 1977) qu'il convient de le mettre en parallèle. Mais les connaissances sur les structures de végétation à l'étage alpin en Amérique du Nord restent trop fragmentaires pour qu'il soit possible de confirmer les affinités "méditerranéennes" des composantes floristiques de ce type de végétation, ce qui semble toutefois au moins localement probable.

\section{Les autres structures de végétation}

Pour compléter la Fig. 2, nous avons été amenés à faire figurer un certain nombre de structures de végétation qui ne s'inscrivent pas a priori, dans la végétation potentielle californienne. II s'agit, de formations forestières zonales septentrionales, de formations steppiques, ou encore de formations azonales.

- Parmi les structures forestières potentielles qui seront seulement évoquées, il convient de citer les suivantes, qui appartiennent toutes à la province nord-ouest pacifique ("Pacific Northwest Floristic Province") :

Les formations à Sequoia sempervirens développées surtout au long de la côte pacifique apparaissent de façon éparse depuis Monterey, puis en peuplements quasi-continus au nord de San Francisco. Elles répondent à un bioclimat perhumide $(P>1300 \mathrm{~mm})$ de type ther- mo-méso-californien très atténué, sans période évidente de sécheresse estivale. Vers le sud Sequoia est associé à Lithocarpus densiflora, Arbutus menziesii et divers Quercus sclérophylles ; plus au nord, apparaissent progressivement Pseudotsuga menziesii, Abies grandis, Tsuga heterophylla, Picea sitchensis, etc. (Zinke, 1977).

Les forêts des Monts Klamath situés en limite nord de la carte correspondent à des structures très complexes (Sawyer et Thornburgh, 1977) et extrêmement riches en essences forestières, mais ne s'intégrant plus de façon évidente à un climat de type méditerranéen. L'horizon inférieur de type collinéen est dominé par $Q$. kelloggii, Q. garryana, Abies concolor, Pseudotsuga menziesii, et l'horizon supérieur (montagnard et subalpin) par Abies magnifica, Tsuga mertensiana, Pinus albicaulis.

- La végétation steppique se développe très largement sur le revers oriental des chaînes montagneuses et répond à deux ensembles principaux :

Les steppes méridionales chaudes à Larrea tridentata essentiellement présentes dans le désert de Mojave mais aussi dans le désert de Sonora.

Les steppes septentrionales froides à Artemisia tridentata, développées sur le revers occidental de la Sierra Nevada, sont représentées par un grand nombre de groupements en fonction des critères édapho-climatiques. Artemisia arbuscula et $A$. nova y jouent également un rôle appréciable.

- La végétation azonale californienne ne sera que partiellement évoquée. En raison des superficies occupées nous signalerons cependant les suivantes :

Les forêts riveraines, encore localement bien représentées dans la vallée centrale, sont dominées par Populus fremontii, Alnus rhombifolia, Platanus racemosa, 
Salix gooddingii, S. laevigata, Acer negundo subsp. californicum. Quercus lobata est également présent.

La végétation hygrophile occupait toute la zone médiane de la vallée centrale où elle est encore bien développée par endroits. Les grandes Cypéracées et Juncacées dominent (Scirpus acutus, S. californicus, $S$. robustus, Carex obnupta, $C$. senta, Juncus effusus, Thypha latifolia, etc.).

La végétation halophile est présente en particulier sur les marges de la baie de San Francisco (formations à Salicornia virginica et Spartina foliosa), et dans les portions arides du sud de la vallée centrale (formations à Atriplex polycarpa, $A$. fruticulosa, A. spinifera, Lycium cooperi, etc.).

\section{Conclusions}

II paraît donc tout à fait possible de proposer, pour la région méditerranéenne californienne, une zonation de la végétation tout à fait superposable à celle qui est le plus souvent utilisée en région circumméditerranéenne. Si certains problèmes méritent d'être précisés, la Fig. 2 illustre schématiquement la répartition des divers étages sur le terrain.

Afin de mieux saisir la signification écologique des principales essences évoquées ci-dessus, il nous a paru utile de faire figurer sur un schéma (Fig. 4) leurs exigences respectives vis-à-vis des bioclimats méditerranéens sensu Emberger (1945), mais aussi des étages altitudinaux de végétation.

Bien évidemment il ne s'agit là que d'une esquisse qu'il conviendra d'affiner, mais aussi d'interpréter en fonction de l'analyse conduite plus haut. En effet, la Fig. 4 a été établie pour l'ensemble de la région californienne, sans tenir compte de la localisation des espèces sur le terrain, et la plupart d'entre elles ne sont présentes que sur une portion, parfois très faible, du territoire envisagé. Compte tenu de ces réserves, ce schéma permet toutefois de proposer une interprétation comparative des exigences des principales essences, en fonction des précipitations et des températures, comme cela a déjà été réalisé en région circum-méditerranéenne (Quezel, 1977), et de saisir de la sorte les analogies écologiques existant entre les principales essences forestières de ces deux régions. Ces informations sont, bien sûr, fondamentales pour l'utilisation à des fins de reforestation des espèces californiennes en région circum-méditerranéenne, compte tenu toutefois des remarques formulées au début de ce travail.

Il convient enfin d'évoquer en terminant, un certain nombre d'homologies et de différences existant entre ces deux régions. Les convergences majeures apparaissent aux niveaux suivants :

- les chênes sclérophylles caractérisent essentiellement le méso-méditerranéen et le méso-californien subhumide et s'étendent, au moins localement, de l'humide (et localement du per-humide) au semi-aride; ils s'infiltrent au thermo-méditerranéen et au thermo-californien. Dans le sud du pourtour méditerranéen, ils colonisent également la majeure partie du méditerranéen supérieur, voire du montagnard méditerranéen, où ils constituent des groupements climaciques; en Californie, présents au californien supérieur, ils y demeurent en revanche très largement dominés par les formations à conifères;

- les chênes caducifoliés ou du moins, certains d'entre eux, s'étendent partout en bioclimat humide et au méditerranéen et au californien supérieurs; la situation est assez comparable entre le Maghreb et la Californie où leur rôle est relativement restreint alors que, sur tout le revers nord de 


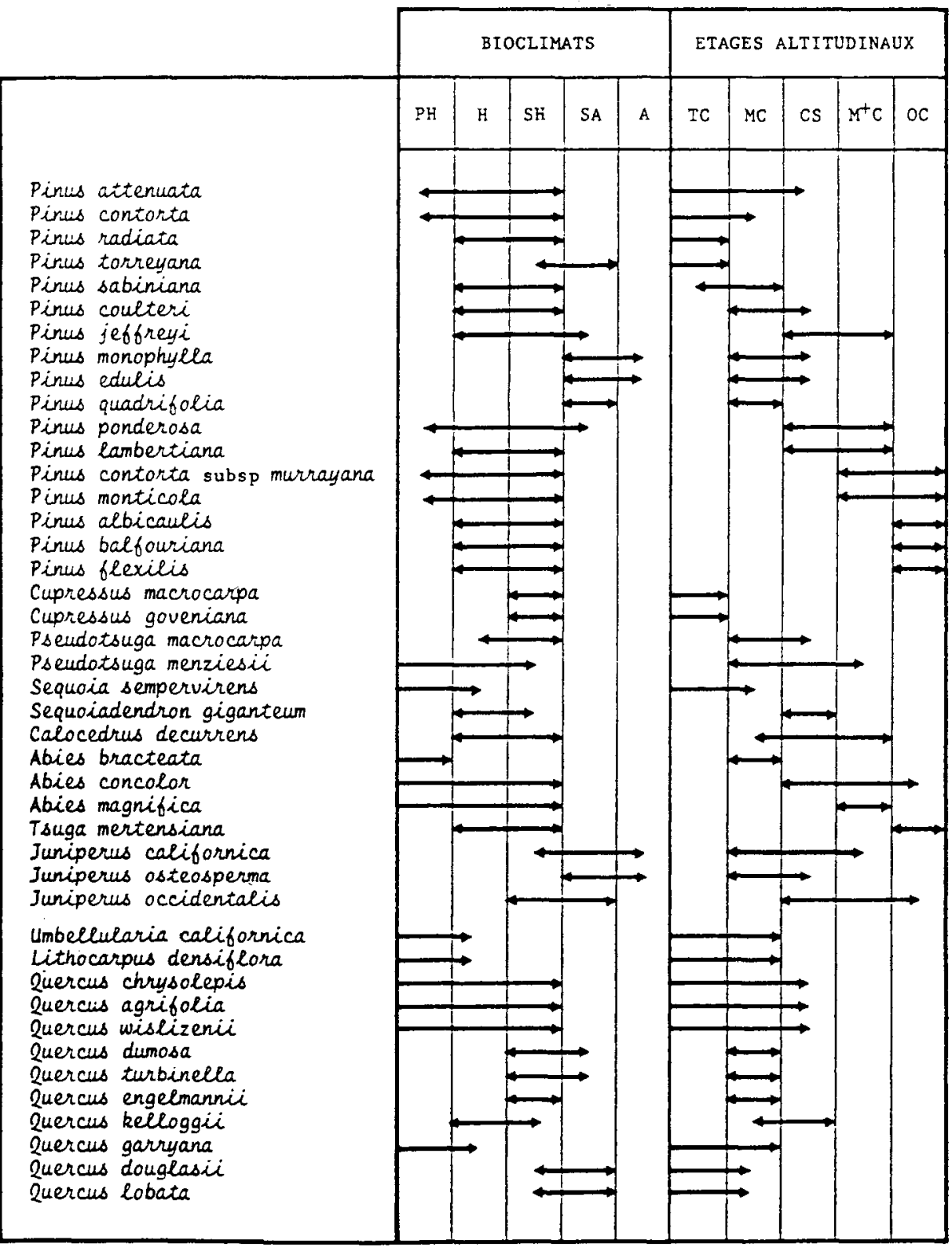

Fig. 4. Signification bioclimatique et altitudinale schématiques, des principales essences forestières de la région méditerranéenne de Californie. PH : Per-Humide, H : Humide, SH : Sub-Humide, SA : Semi-Aride, A : Aride, TC Thermo-Californien, MC : Méso-Californien, CS : Californien Superieur, MtC : Montagnard Californien, $O C$ : Oro-Califomien. 
la Méditerranée, leur extension est bien plus large aussi bien du point de vue altitudinal que bioclimatique;

- les conifères thermophiles (Pinus, Cupressus) forment, théoriquement au moins, des groupements climaciques au thermo-méditerranéen et au thermo-californien. Ils s'étendent également en structures paraclimaciques au méso-méditerranéen et californien semi-aride et subhumide;

- Les conifères d'altitude constituent généralement des structures climaciques à partir du montagnard. Toutefois cette situation est surtout confirmée en Californie puisqu'en Europe, Fagus silvatica et surtout les Quercus caducifoliés sont largement présents en bioclimat per-humide et humide, alors qu'au sud de la Méditerranée les chênes sclérophylles dominent au subhumide et au semi-aride;

- les représentants du genre Juniperus jouent un rôle important en bioclimat semiaride aussi bien en Méditerranée qu'en Californie.

Parmi les différences, certaines sont capitales, alors que d'autres restent de signification plus limitée. Parmi les premières il convient de citer :

- la présence en certaines parties de la Californie, de formations climaciques à Quercus caducifoliés au thermo-californien semi-aride ( $Q$. douglasii) qui ne possèdent pas de correspondant en région circum-méditerranéenne (sauf peut-être, très localement, les structures semi-caducifoliées du Proche-Orient à $Q$. ithaburensis);

- l'existence en Californie, comme d'ailleurs en d'autres régions de l'Amérique du Nord, de formations à Pinus à grosses graines («pinyons») qui constituent un climax fréquent, associés à des Juniperus arborescents voire à des Cupressus, en bioclimat semi-aride;
- la signification de la "mixed evergreen forest" pose également problème et aucune structure particulière de végétation peut a priori lui être mise en parallèle en région circum-méditerranéenne. Cela peut être lié à des raisons historiques, mais au moins en partie, à la rareté, voire l'absence dans l'ancien monde de situations écologiquement comparables (per-humide chaud et tempéré surtout). Dans ce cas, les chênes sclérophylles ou caducifoliés peuvent être présents, mais ils ne constituent pas de structures de végétation originales. En fait, comme nous l'avons suggéré plus haut c'est certainement avec la laurisylve canarienne que cette végétation peut être le plus valablement comparée.

Dans le second groupe de différences, signalons les plus significatives :

- les chênes sclérophylles qui, rappelonsle, ne sont en aucune façon exclusifs du climat méditerranéen en Amérique du Nord, sont représentés en Californie par un nombre plus élevé d'espèces (10 contre 5). La situation est inverse pour les chênes caducifoliés et semi-caducifoliés (4 contre plus d'une douzaine);

- les conifères thermophiles, bien que numériquement: moins bien représentés en région circum-méditerranéenne (respectivement 6 et 10 contre 3 et 2 Pinus et Cupressus, mais aussi le genre monospécifique Tetraclinis), $y$ jouent un rôle infiniment plus important dans la constitution des paysages végétaux. La plupart des espèces californiennes n'occupent en effet que des territoires exigus;

- les conifères d'altitudes sont beaucoup plus nombreux en Californie. Les genres Sequoia, Sequoiadendron, Pseudotsuga, Tsuga, Libocedrus font en particulier défaut dans la région circum-méditerranéenne, qui ne possède en propre que le genre Cedrus. De même, leur place dans le paysage végétal y est beaucoup plus importante et ils forment à peu près par- 
tout des groupements climaciques, notamment au californien supérieur, alors qu'à l'étage correspondant autour de la Méditerranée, les chênes occupent une situation prépondérante. En fait, seuls quelques Abies (A. cephalonica, A. pinsapo, $A$. maroccana) y jouent un rôle comparable mais toujours limité géographiquement. De même, en Californie, les Pins sont nombreux, alors qu'en région circumméditerranéenne le groupe Pinus nigra est pratiquement seul, mais représenté par plusieurs sous-espèces vicariantes;

- l'étage oro-californien présente également un nombre élevé d'espèces où dominent les représentants du genre Pinus alors que dans l'ancien monde, seuls Pinus heldreichii, Juniperus thurifera et $J$. excelsa jouent un rôle analogue.

\section{Références}

Ackerman E.-A. (1941) The Köppen classification of climates in North America. Geogr. Rev. $31,105-111$

Akman Y., Barbero M. \& Quezel P. (1978) Contribution à l'étude de la végétation forestière d'Anatolie méditerranéenne. Phytocoenologia, 5 (1), 1-79

Aschmann $\mathrm{H}$. (1973) Distribution and peculiarity of Mediterranean ecosystems. In : Mediterranean Type Ecosystems, Ecological Studies 7 (F. Di Castri and H.A. Mooney Editors), Springer-Verlag, Berlin, pp. 11-19

Aschmann $H$. (1984) A restrictive definition of mediterranean climates. Bull. Soc. Bot. France, 131, Act. Bot. 2/3/4, 21-30

Bagnouls F. \& Gaussen H. (1953) Saison sèche et indice xérothermique. Doc. Cartes Product. végétales, Ser. Gen. 3, 8, 1-48

Barbero M. \& Quezel P. (1981) Methods of classifying ecosystems in the mediterranean rim countries and in the South-Western. U.S.A. Symp. on Dynamic and Management of Mediterranean-type ecosystems, San Diego, California

Barbero M., Quezel P. \& Rivas-Martinez S. (1981) Contribution à l'étude des groupements forestiers et préforestiers du Maroc. Phytocoenologia, 9 (3), 311-412

Barbour M.-G. \& Major J. (1977) Terrestrial vegetation of California. J. Wiley and Sons, New York

Clements F.-E. (1920) Plant indicators. Carnegie Inst. Wash. Publ. 290, 1-388

Cooper W.-S. (1922) The broad-sclerophyll vegetation of California. Carnegie Inst. Wash. Publi. 319, 1-124

Daget P. (1977) Le bioclimat méditerranéen : Analyse des formes climatiques par le système d'Emberger. Vegetatio, 34, 2, 87-104

Emberger L. (1930) La végétation de la région méditerranéenne. Essai d'une classification des groupements végétaux. Rev. Gen. Bot., 42, 641-662, 705-721

Emberger L. (1945) Une classification biogéographique des climats. Recueil Trav. labot. Geol. Zool. Fac. Sci. Montpellier, Bot. 7, 3-43

Flahault C. (1901) introduction. In Flore de France, Lechevalier Paris, $52 \mathrm{pp}$.

Gaussen H. (1926) Végétation de la moitié orientale des Pyrénées. Lechevalier, Paris, 526 pp.

Griffin J. (1977) Oak woodland. In (Barbour et Major), 383-416

Hanes T. (1981) California chaparral. In : Ecosystems of the world (Di Castri ed.) II, Elsevier, pp. 139-174

Harvay Th. (1980) Environmental factors in Giant Sequoia. Ecology : 13-40 U.S. Depart. of the Interior Nat. Park Serv., Sc. Series np 12, Washington DC.

Heady H.-F. et al. (1977) Coastal prairie and northern, coastal shrub. In Terrestrial Vegetation of California (Barbour et Major ed.) Wiley and Sons, New York, 733-762

Heady H.-F. (1977) Valley grassland. In Terrestrial Vegetation of California (Barbour et Major, ed.) Wiley and Sons, New York, 491-514

Koppen W. (1936) Das Geographische System der Klimate, vorzugsweise nach ihren Beziehungen zur Pflanzenwelt. Geograph. Zeischrift, 6. 1-54

Major J. \& Taylor W. (1977) Alpine. In Terrestrial Vegetation of California (Barbour et Major, ed.) Wiley and Sons, New York, 601-675

Mooney H. (1977) Southern coastal scrub. in Terrestrial Vegetation of California (Barbour et Major, ed.) Wiley and Sons, New York, 471490 
Ozenda P. (1975) Sur les étages de végétation dans les montagnes du bassin méditerranéen. Doc. Cart. Ecol. 16, 1-32

Quezel P. (1974) Les forêts du pourtour méditerranéen. U.N.E.S.C.O, Programme "Homme et Biosphère". Com. Nat. Fr. Mab. 1-53

Quezel P. (1979) La région méditerranéenne française et ses essences forestières, signification écologique dans le contexte circum-méditerranéen. Forêts Méditerranéennes, 1, 1, 7-18, Marseille

Quezel P. (1979) «Matorrals" méditerranéens et "chaparrals" californiens, quelques aspects comparatifs de leur dynamique, de leurs structures et de leur signification écologique. Ann. Sci. For., 36, 1, 1-12

Quezel P. (1981) Floristic composition and phytosociological structures of sclerophyllous matorrals around the mediterranean. In : Ecosystems of the world, II (Di Castri ed.) Elsevier, pp. 107-122

Quezel P. \& Bonin G. (1980) Les forêts feuillues du pourtour méditerranéen, constitution, écologie, situation actuelle, perspectives. Rev. For. Fr., XXXII, 3, 253-268

Quezel P. \& Barbero M. (1981) Contribution à l'étude des formations présteppiques à Genévriers au Maroc. Bull. Soc. Broteriana, Ser. 2, $1137-1160$

Quezel P. \& Schevock J. (1982) Essai de mise en parallèle de la zonation des structures forestières de végétation entre la Californie méridionale et le pourtour méditerranéen. Ecologia Mediterranea, VIII, 1-2, 15-29, Marseille

Quezel P. \& Taylor H.-C. (1984) Problèmes posés par les relations climat-dynamique de la végétation dans les régions méditerranéennes de l'ancien monde, du Cap et de Californie. Bull. Soc. Bot. Fr., 131. Act. Bot. 2/3/4, 235-245

Quezel P. \& Barbero M. (1985) Carte de la végétation potentielle de la région méditerranéenne. 1 : Méditerranée Orientale. C.N.R.S., Paris, 69 pp. + carte

Raven P. et Axelrod D. (1978) Origin and relationships of the Californian Flora. Univ. Calif. Public. Botany, 72, Berkeley and Los Angeles, $134 \mathrm{pp}$.
Rivas-Martinez S. \& Arnaiz C. (1984) Bioclimatologia y vegetacion en la Peninsula iberica. Bull. Soc. Bot. France, 131. Act. Bot. 2/3/4 : $111-120$

Rundel P., Parsons D. \& Gordon D. (1977) Montane and subbalpine vegetation of the Sierra Nevada and cascade ranges. In Terrestrial Vegetation of California (Barbour et Major, ed.) Wiley \& Sons, New York, 559-599

Santos-Guerra A. (1984) Clima mediterraneo de Canarias y vegetacion. Bull. Soc. Bot. Fr. , $131,2 / 3 / 4,121-128$

Sawyer J.-O. \& Thornburgh D. (1977) Montane and subalpine vegetation of the Klamath mountain. In Terrestrial Vegetation of California (Barbour et Major, ed.) Wiley \& Sons, New York, 699-731

Sawyer J., Thorriburgh D. \& Griffin R. (1977) Mixed evergreen forest. In Terrestrial Vegetation of California (Barbour et Major, ed.) Wiley \& Sons, New York, 359-381

Snow G. (1972) Some factors controlling the establishment and distribution of Quercus agrifolia and Q. engelmannii in certain southern californian oak woodlands. Ph. D. diss. Oregon State Univ., Corvallis, 105 pp.

Thorne R. (1977) Montane and subalpine forest of the transverse and peninsular ranges, In Terrestrial Vegetation of California (Barbour et Major, ed.) Wiley \& Sons, New York, 537-558

Vogl R. et al. (1977) The closed cone-pines and Cypresses. In Terrestrial Vegetation of California (Barbour et Mlajor, ed.) Wiley \& Sons, New York, 295-356

Walter H., Harnickell E. \& Mueller-Dombois D. (1975) Climate-diagram. Maps Suppl. to the vegetation monographs, Springer-Verlag, Berlin Heidelberg and New York

Young J., Evans R. \& Major J. (1977) Sagebrush steppe. In Terrestrial Vegetation of California (Barbour et Major, ed.) Wiley \& Sons, New York, 763-795

Zinke P.-J. (1977) The redwood forest associated north-coast forest. In Terrestrial Vegetation of California (Barbour et Major, ed.) Wiley \& Sons, New York, 679-697 